\title{
Rethinking structural masonry: unreinforced, stone-cut shells
}

1 Matthias Rippmann Dipl-Ing PhD candidate, Institute of Technology in Architecture, ETH Zurich, Switzerland
(2) Philippe Block MSc, SMArchS, PhD

Assistant Professor, Institute of Technology in Architecture, ETH Zurich, Switzerland
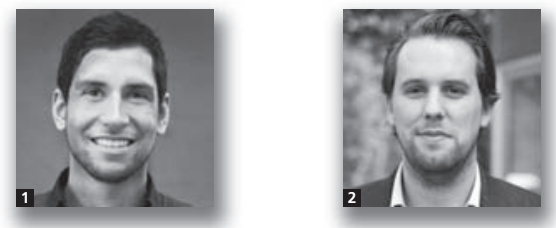

Recently, the interest of architects and designers in contemporary applications of masonry has increased considerably. Motivated by the elegance of historic masonry structures, mostly decorative stone and brick applications have been developed, driven by new possibilities in fabrication technology and the increasing relevance of sustainable building materials. In contrast, the use and potential of structural masonry has rarely been addressed in these developments. This paper presents novel methods for structural stone masonry, focusing on the possibilities of approaches closely interrelating form-finding and material-driven fabrication. Thanks to newly developed structural form-finding methods for the design of unreinforced masonry shells, new, 'free-form' vaulted structures in stone are now imaginable. These new structural design tools have been integrated into a digital process, which is informed by relevant construction and fabrication parameters. The paper focuses on this interrelation, linking expressive structural form to its real-world demands in stone construction by considering appropriate and efficient fabrication technology. The design for the MLK Jr Park Vault in Austin, Texas, USA is used as a proof-of-concept case study for the process, taking full advantage of modern stone-cutting technology and using the compression strength and weight of stone masonry to efficiently combine construction material and structural form.

\section{Introduction}

The resistant virtues of the structure that we seek depend on their form; it is through their form that they are stable, not because of an awkward accumulation of material. There is nothing more noble and elegant from an intellectual viewpoint than this: to resist through form. (Eladio Dieste, 1996)

'Good structural form' results in low compressive stresses and reduces the need for bending capacity of a structure, regardless of what material has been chosen. The potential of funicular structures is demonstrated in the most elegant way by the use of unreinforced stone in Gothic vaults. Recently developed threedimensional equilibrium analysis methods make it possible to explain how these stunning historic masonry vaults stand, and, by learning from these engineering masterpieces, to explore novel forms for this old material (Block and Ochsendorf, 2007). These new form-finding approaches offer surprising possibilities for formal expression, which at the same time addresses today's requirements for sustainable and resource-efficient construction.
A renewed interest in innovative architectural applications for stone, and masonry in general, led to several unique prototypical structures in recent years. An iconic example is the Mapungubwe Interpretive Centre in South Africa (Ramage et al., 2010a, 2010b). The use of a predominately local building material (in situ soil-pressed, cementstabilised tiles) in combination with traditional tile vaulting addresses the skills and needs of the local communities through capacity building and safe technology transfer (Block et al., 2010a), and takes into account limitations of the remote building site. Another example is the 'free-form' vault prototype built at ETH Zurich in 2011 (Davis et al., 2012).

Note that in this context, and adopted throughout the paper, the term 'free-form' refers to the complexity of the double-curved, often unexpected forms of compression-only structures.

Several other projects have combined the use of stone or brick with state-of-the-art digital design and fabrication techniques 
(Bechthold, 2009; Fallacara, 2009; Gramazio and Kohler, 2008; Kaczynski et al., 2011; Pedersen et al., 2012; Wendland, 2009). However, the design for the majority of these projects was driven by visual, tectonic and ornamental considerations. Indeed, structural analysis tools were used to verify the structural performance of these designs, but only few have addressed or have fully exploited the unique structural capacity of masonry structures in compression by integrating structural form finding in the design process.

The research presented in this paper focuses on strategies for innovative and efficient, unreinforced, stone-cut vault design for contemporary architectural applications, based on novel digital tools and the latest industrial fabrication technology. It enters the relatively new research field of digital stereotomy (Fallacara, 2006, 2009). Digital stereotomy revisits and extends traditional stereotomy, the art of cutting up stone in discrete blocks (Fitchen, 1981) by introducing computational strategies for the design, digital fabrication and installation of the complex stone blocks.

A key aspect is to define and develop a suitable and coordinated design and fabrication set-up for the production of the hundreds of individual voussoirs that need to be processed for a single vault design. Owing to the threedimensional shape of the separate blocks and the geometrically complex fabrication constraints, the challenge is to coordinate the design of the individual voussoirs, in accordance with the technical machine set-up. Furthermore, the right balance needs to be found between form finding and fabrication constraints, in order to produce free-form stone vaults efficiently. The potential of a well-coordinated digital chain for realising complex stone vaults has not been exploited yet, because most contemporary research in digital masonry and stone has been focusing on the ornamental possibilities.

In particular, this paper describes the main workflow for the design and materialisation process of the MLK Jr Park Vault Project in Austin, Texas, USA. The research is driven by the fascination for the elegance of Gothic stone vaults that combine aesthetics, ornamentation and structural logic, as well as by the ambition to breathe new life into an apparently obsolete building technique. By fully embracing new funicular form finding and structurally informed fabrication optimisation strategies, the authors strongly believe that new forms in structural masonry can enrich the vocabulary of contemporary architecture.

This paper is structured as follows. The next section introduces the general process, describing the digital design and materialisation chain from form finding to materialisation, and listing the driving fabrication constraints. In Section 3, the implemented structural design approach and the structurally and fabrication informed design of the stereotomy (tessellation and voussoir geometry) are described in detail. Section 4 then illustrates how these methods were used for the design development of the MLK Jr Park Vault. Finally, Section 5 discusses the results and sets out future research.

\section{Digital design and materialisation chain}

This section describes the sequential, but interrelated steps of the digital chain from form finding to materialisation of freeform stone vaults.

\subsection{Overview}

Figure 1 shows the steps of the process and their interdependencies. The steps are categorised in three main phases: design process, analysis process and materialisation process.

The first phase of the digital chain is the design process, which consists of three steps. The defining structural properties for stone, or masonry in general, are its low tensile and high compressive strength. Because of this, to span space in unreinforced masonry, the use of funicular form, acting purely in compression, is mandatory to ensure structural stability. Therefore, in the first step, an appropriate funicular form is determined (Section 3.1). In the second step, based on the results of the funicular form finding, a possible tessellation geometry is generated that defines the cutting strategy of the vault. This is an automated process, informed by structural and fabrication-related data, which can be influenced or guided by the designer (Section 3.2). In the third step of the design process, the tessellation pattern is used to generate the voussoir geometry considering structural as well as fabrication and assembly constraints (Section 3.3).

In the second phase, the results of the design process are verified using inverse equilibrium analysis (Block and Lachauer, 2011), structural models (Block et al., 2010b; Van Mele et al., 2012) and discrete-element modelling (DeJong, 2009). Based on the output of this structural analysis phase, the design is refined, if necessary.

In the third and last phase, the components of the structure are fabricated and installed using the machine set-up that defined the constraints for the design process.

Figure 2 gives an overview of the constraints on the design process, which can be grouped into

(a) architectural and tectonic requirements (Section 2.2)

(b) structural requirements (Section 2.3)

(c) fabrication and installation requirements (Section 2.4).

In the following sections, these three groups of constraints are discussed in more detail. 


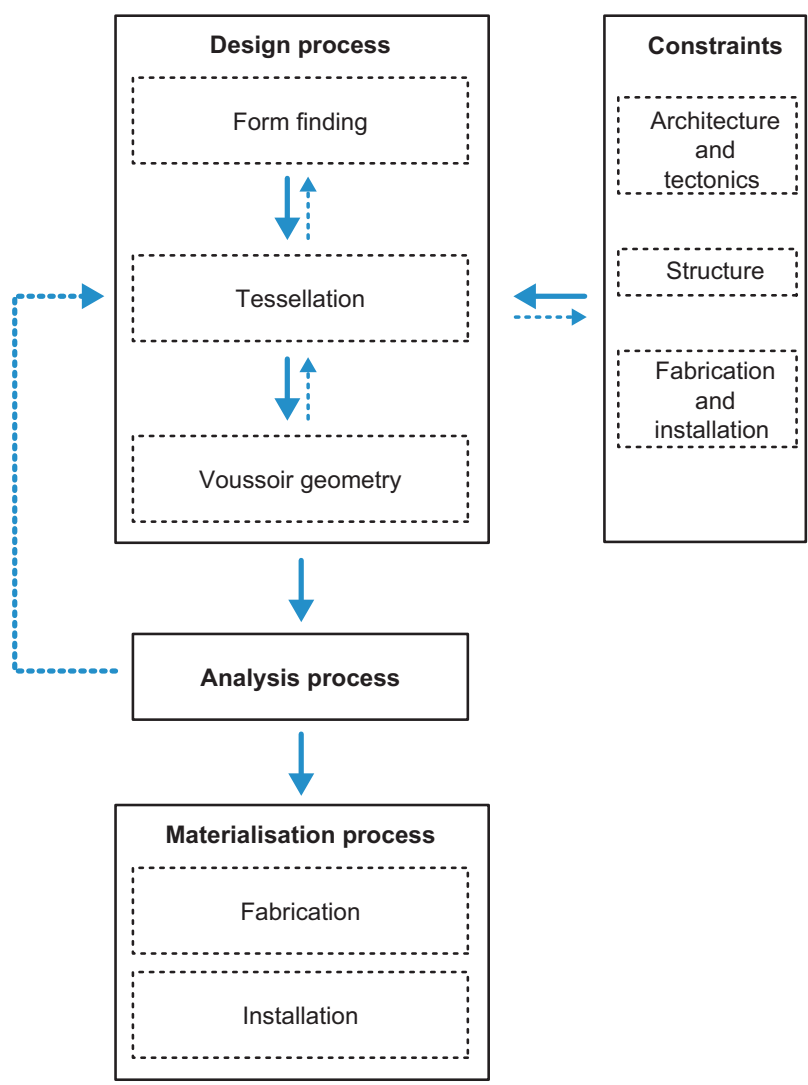

Figure 1. Flow diagram of the design, structural analysis and materialisation phases of discrete, free-form stone vaults showing the interdependencies with relevant constraints

\subsection{Architectural and tectonic requirements}

From all aspects that influence the desired overall shape of the vault, the architectural and tectonic intents, which include contextual, functional and visual considerations, are the least restrictive ones.

Even though the shape generation has to follow structural requirements, structural design approaches have been implemented in the presented digital chain that allow balancing of the constraints of structural form with the designer's intents, by giving the designer careful and explicit control over all parameters of the form finding (Section 3.1).

\subsection{Structural requirements}

The thickness of the vault and thus the local offset values for the voussoirs generation should be sufficient to provide stability under live loading and to reduce the danger of buckling. The ideal orientation of the tessellation is aligned to the local force vector field (Figure 3(a)), which is obtained from the results of the thrust network analysis (TNA) form finding (Figure 3(b)). Therefore, the voussoirs' contact faces

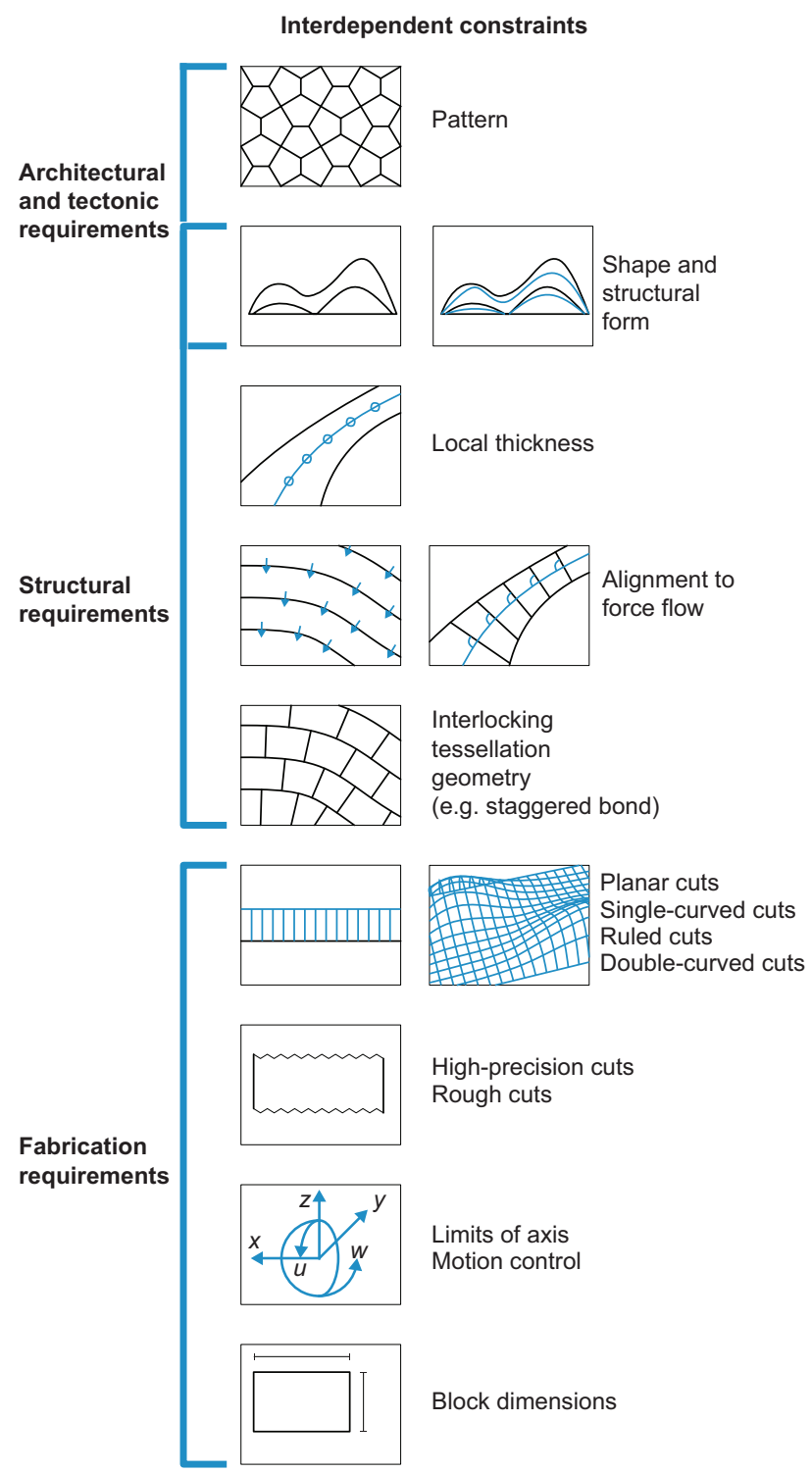

Figure 2. Interdependent constraints to be considered in the design of free-form stone vaults, grouped in architectural and tectonic, structural and fabrication requirements

should be aligned as perpendicular (Figure 3(d)) and parallel (Figure 3(e)) as possible to the force flow to prevent sliding failure between them.

To have load-transmitting contact faces between voussoirs that are orthogonal to the force flow, the thrust surface normals along the edges of the tessellation are used to construct the voussoir faces (Figure 3(c)). These are thus ruled surfaces; this means that they can be described by a moving straight line. Furthermore, as an additional measure to avoid local sliding failure, the minimal and maximal overlaps between voussoirs 


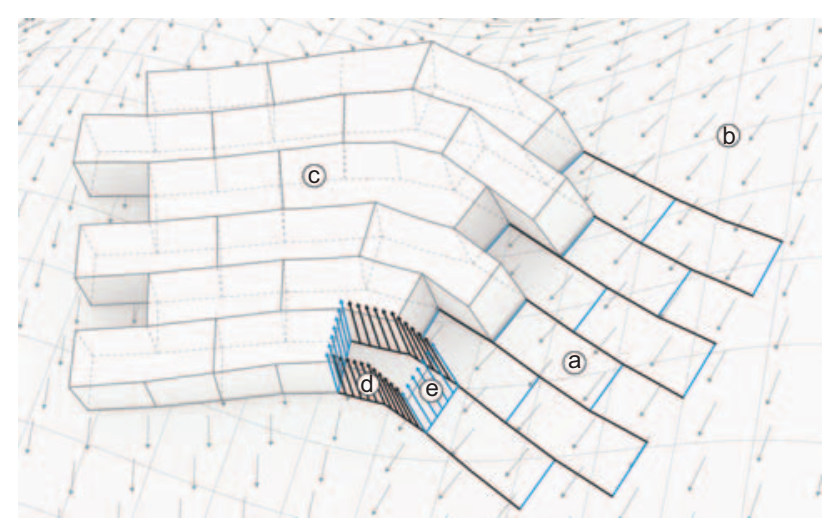

Figure 3. Relation between (a) the tessellation geometry, which is based on (b) the force vector field and (c) the voussoirs with contact faces (d) perpendicular and (e) parallel to the force flow

should be controlled. A good stereotomy provides the necessary interlocking between blocks such that they form a stable three-dimensional structural surface. This strategy creates, for example, a staggered bond as shown in Figure 3.

\subsection{Fabrication requirements}

In contrast to most building construction materials, natural stone demands a subtractive process for its fabrication. Subtractive processes tend to be inherently less resourceefficient because more material needs to be processed than the amount contained in the end product. Therefore, one aspect of the research is the development of efficient strategies for the machining of complex building parts in stone that take into account material waste, tool degradation and cutting time. Another aspect is to consider how fabrication requirements determine geometrical constraints for the design process (Pigram and McGee, 2011).

Computer numerically controlled (CNC) machines are widely used in the stone-cutting industry (Garrido Campos and Marín Martín, 2010). Each type of machine and tool configuration has its advantages and disadvantages, varying in terms of cost and efficiency, accuracy, quality of the surface finish, and the type of shapes they are able to produce. Processing stone is a complex task in which, in order to obtain the most economic cutting conditions, the ideal balance has to be obtained between cutting technique, tool life, cutting rate, tolerance and quality. Three commonly used types of $\mathrm{CNC}$ stone-cutting machines for complex geometry are

multi-axes abrasive water jets

multi-axes diamond wire cutters

multi-axes milling and circular saw blade machines.

Water jets use a high-velocity and high-pressure jet of water and abrasive substance to cut through the material. There is no heat generation during the cutting process, and tolerances and material waste are very low. However, depending on the material, the depth of the cut is limited to $50-150 \mathrm{~mm}$, which makes water jet technology unsuitable for cutting larger stone blocks.

Wire cutters are mostly used as block-cutting machines for the primary sawing of blocks into slabs or the pre-cutting of larger pieces before further, more refined processing. However, fouror six-axes diamond wire cutters can be used to process complex geometry based on ruled surfaces (Rippmann and Block, 2011). Depending on the material and wire used, the tolerances of the cuts tend to be insufficient for complex geometry, which is needed for the current purposes.

For processing complex geometry in stone, five- or six-axes milling and circular-saw-blade machines are most popular (Garrido Campos and Marín Martín, 2010). Usually, these machines have a portal design, capable of holding different milling heads and circular saw blades. This offers a flexible setup for accurate subtractive stone milling and cutting. Using milling heads for cutting stone layer by layer results in very precise surfaces with total geometric freedom, but comes at the cost of relatively high amounts of waste material, low cutting rates and fast tool degradation. The use of circular saw blades, on the other hand, minimises waste material, cutting time and tool degradation, but limits the movement of the blade in the stone to planar cuts. However, progressive cutting strategies allow for free-form geometries to be cut (see Section 4.4).

Geometrical limitations and the machine set-up need to be specified, balancing the technical feasibility of the machine process and the geometrical flexibility needed. In particular, the limits of axis motion need to be addressed in the design process in order to obtain sufficient geometric flexibility to process specific voussoir geometries. This is related to the minimum and maximum dimension and volume of the voussoirs, which are defined by the physical limitations of fabrication and assembly, and by the practical limit of block sizes that can be handled on site during assembly.

\section{Design process}

As pointed out in Section 2, the design process phase contains three sequential but interdependent steps: form finding, tessellation and voussoir geometry. This section describes these steps, considering the fabrication constraints and requirements addressed in the previous section. The goal is to identify efficient ways to achieve a feasible construction.

\subsection{Form finding}

The form finding in the present authors' research is based on TNA, which is a graphic statics-based approach to the equilibrium design and analysis of compression-only vaulted 
structures with complex geometry. The method uses geometrically linked form and force diagrams, which can be manipulated by the designer explicitly to control or steer the funicular form finding (Block and Ochsendorf, 2007; Block, 2009; Rippmann et al., 2012), or which can be optimised in an automated fitting procedure to approximate a given target surface (Block and Lachauer, 2011).

As a short introduction, Figure 4 depictures the basic, graphical components of the form-finding method: a form diagram $\Gamma$, defining the geometry of the structure and the layout of forces in plan; two possible corresponding force diagrams $\Gamma_{1}^{*}$ and $\Gamma_{2}^{*}$, representing and visualising two possible distributions of horizontal thrust; and $\mathrm{G}_{1}$ and $\mathrm{G}_{2}$, the corresponding thrust networks in equilibrium with given (vertical) loading.

A continuous thrust surface can be fitted through the nodes of the obtained thrust networks, and the 'flow of forces' in the vault can be visualised as a vector field. This field provides an alternative representation of the equilibrium of the vault that is more useful for the applications in this paper, since it provides almost continuous, that is topology-independent, information of the force equilibrium.

\subsection{Tessellation}

As described in Section 2.4, the tessellation geometry needs to be laid out on the thrust surface such that edges are orientated as perpendicular or parallel as possible to the local force vectors. At the same time, bounds on the edge lengths need to be imposed because of fabrication constraints. The tessellation furthermore needs to have an 'interlocking bond' to allow for fully three-dimensional structural action, and to prevent sliding of individual voussoirs. To deal with these hard-to-control, interrelated criteria, the authors developed an optimisation scheme that simplifies the design of appropriate tessellation
$\Gamma$

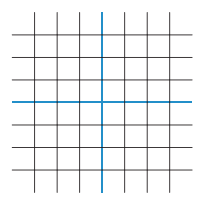

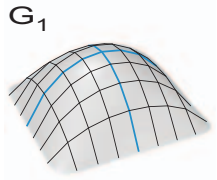

$\Gamma_{1}^{*}$

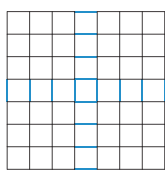

$\mathrm{G}_{2}$

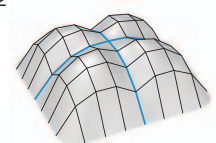

$\Gamma_{2}^{*}$

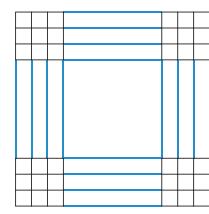

Figure 4. Thrust network analysis: form diagram $\Gamma$, two possible force diagrams $\Gamma_{1}^{*}$ and $\Gamma_{2}^{*}$, and the corresponding thrust networks $\mathrm{G}_{1}$ and $\mathrm{G}_{2}$ for a given (vertical) loading geometries for free-form vaults. Implemented in a digital design tool, it offers an interactive, flexible and user-driven design process, regulated and monitored automatically in real time.

The topology of the tessellation is defined by drawing lines onto the thrust surface. These lines and the force vector field, defined at the nodes of the thrust network, are the starting point of the automated process (Figure 5(a)). The edges are classified as perpendicular (black) or parallel (grey) according to their initial direction with respect to the local force flow. Edge lengths can be constrained to specific values or within a given range to incorporate fabrication requirements. Edges to be subjected to these constraints can be selected individually by the user or automatically according to the aforementioned classification.

The optimisation process then proceeds with an iterative twostep solving algorithm that converges towards an equilibrium state at which all edges are as perpendicular or parallel as possible to the local force flow. The basic steps to find this equilibrium are described below.

- Step 1: Each edge is separately aligned based on the given force vector field (Figure 5(a)), using its midpoint as local reference and centre of rotation for the interpolated target vector for that edge. Depending on the classification, each edge is automatically oriented perpendicular or parallel with respect to the target vector, and, if needed, scaled according to edge length limitations. This procedure enforces the correct orientation of all edges individually but results in a disconnected set of lines (Figure 5(a)).

- Step 2: The connectivity and initial topology of the tessellation is restored. This is achieved by identifying previously connected edges and merging their end nodes back into a single node using their barycentre (Figure 5(b)). Owing to the three-dimensional thrust surface, this barycentric node needs to be projected normal onto the thrust surface to guarantee that the tessellation geometry remains on the surface during the iterative process.

Figure 5(c) visualises the iterative procedure, which repeats the two steps described above until all edges are parallel and perpendicular to the local force flow, within a given tolerance. For more complex topologies, competing parameters demand additional stopping criteria such as the maximum number of iterations or the minimal node displacement between successive steps.

The edge length limitations can be used to parametrically change the tessellation to control its staggered and interlocking behaviour. Figure 6 shows three different tessellation geometries based on the same topology. The only varying parameter 


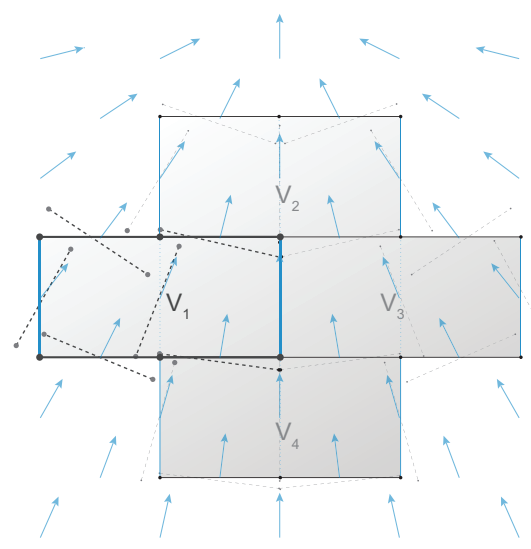

(a)

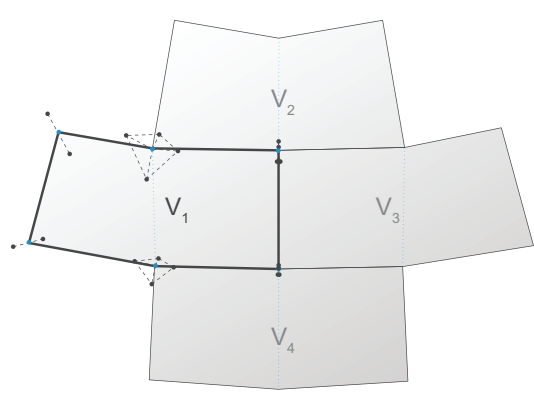

(b)

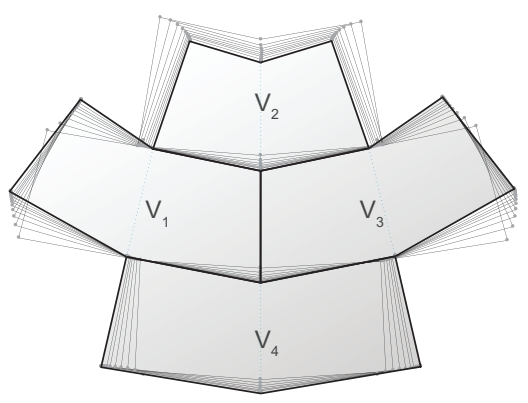

(c)
Figure 5. Iterative alignment procedure: (a) step 1: initial tessellation pattern with aligned line segments (dotted lines) of a polygon $\mathrm{V}_{\mathrm{n}}$ with respect to the local forces. (b) Step 2: based on the end points (black) of the aligned line segments, updated coordinates of the nodes are defined. (c) The updating of the coordinates for each node are processed iteratively is the length of the vertical dotted edges, allowing the control of the cell geometry from a convex hexagon to a dovetail-shaped hexagon. To have a better bond between the voussoirs, the dovetail-shaped cells were chosen (Figure 6(c)). This tessellation furthermore locks the half pieces along unsupported edges (highlighted), preventing them from sliding out (Figure 6(f)). It can be seen that slight topological changes were needed to avoid heavy distortion and size variation of cells.

\subsection{Voussoir geometry}

Individual voussoirs are generated from the tessellation, the thrust surface representing the central axis of the vault and data regarding the local thickness of the structure. The thickness is calculated based on non-funicular live load cases (Allen and Zalewski, 2010). Each contact face is described by lofting through a set of lines normal to the thrust surface to obtain faces aligned normal and tangent to the force flow. In

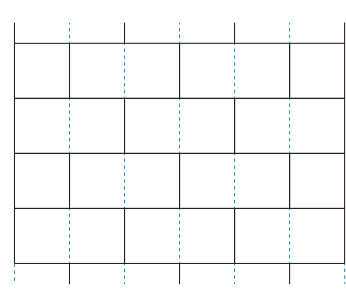

(a)

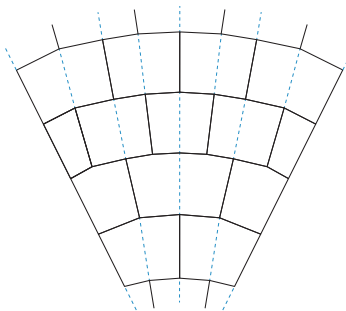

(d)

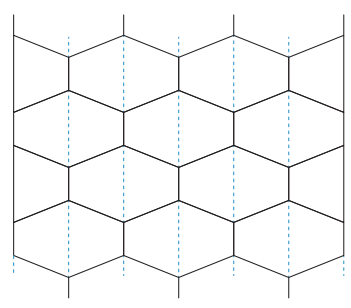

(b)

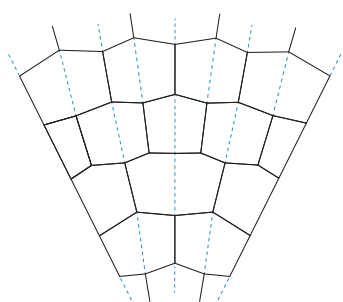

(e)

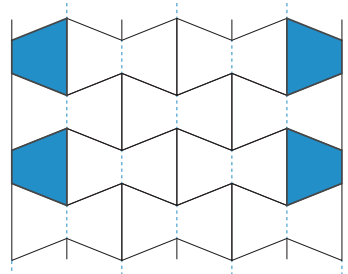

(c)

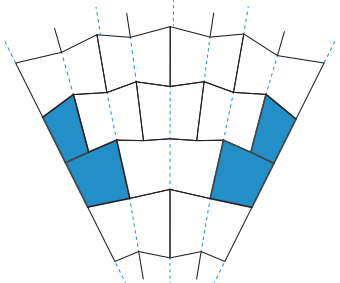

(f)

Figure 6. (a-c) Three tessellations possible with the same topology resulting in different staggered bonds; $(\mathrm{d}-\mathrm{f})$ its configuration in a converging layout highlighting the locked half pieces along the unsupported edge 
the case of free-form vaults the resulting contact faces are twisted ruled surfaces (Rippmann and Block, 2011).

As the ambition is to build dry stone, that is without mortar, the load-transmitting contact faces between voussoirs demand a high geometric accuracy in the fabrication process. Circularblade stone cutting satisfies these precision requirements and is one of the most efficient stone fabrication processes. The upper and lower surfaces of the voussoirs do not require such strict constraints.

Owing to the double-curved geometry of the free-form vaults, the contact faces are not necessarily planar. However, with the selected cutting technology only planar cuts can be made. Therefore, an iterative algorithm was developed to planarise the contact faces. As initial step for the planarisation procedure, each contact face $\mathrm{F}_{n}$ is defined by four nodes, based on the tessellation edges, the local thickness of the vault and the corresponding surface normal $\mathrm{N}_{n}$ (Figure 7(a)).

Next, the following two steps are performed at each iteration until all contact faces are planar within a given tolerance.

Step 1: The best-fit plane $\mathrm{P}_{n}$ is computed using least-squares fitting through the corresponding nodes of the contact faces $\mathrm{F}_{n}$ (Figure 7(b)). The coordinates of each node are updated by normal projection onto its corresponding best-fit plane $\mathrm{P}_{n}$. As a result, neighbouring contact faces are no longer intersecting in a line, which causes detached neighbouring faces.

- Step 2: The connectivity of neighbouring faces is restored by merging face nodes of corresponding faces into a single node at their barycentre (Figure 7(b)). Additionally, the distance between the corresponding intrados (lower) and extrados (upper) barycentre is controlled with respect to the local surface normal, to maintain the required local thickness of the vault during the iterative process.

Figure 7(c) visualises the iterations of this algorithm.

\section{Case study: MLK Jr Park Vault}

This section describes the application of the digital chain discussed in this paper on a large-scale project currently being designed for Austin, Texas, USA. The radical stone structure will be located at the heart of the Martin Luther King (MLK) Jr Park of the East-Austin urban development and transitoriented district Chestnut Plaza, where it will be used as a multi-purpose community and performance space. The vault will cover an area of approximately $600 \mathrm{~m}^{2}$ with a maximum span of $28 \mathrm{~m}$.

\subsection{Form finding - structural design}

The form finding was done with RhinoVault (see www.block. arch.ethz.ch/tools/rhinovault), an implementation of the TNA approach in Rhinoceros (Rippmann et al., 2012). Form and force diagrams of 1094 edges were defined and manipulated to find a resulting thrust network close to the initial sketch design. The thrusts in each edge of the form diagram (Figure 8(a)), hence the thrust network (Figure 8(c)), can directly be measured from the force diagram, which is drawn to scale (Figure 8(b)). For example, the horizontal forces due to selfweight are the highest in the edges of the lower right support in

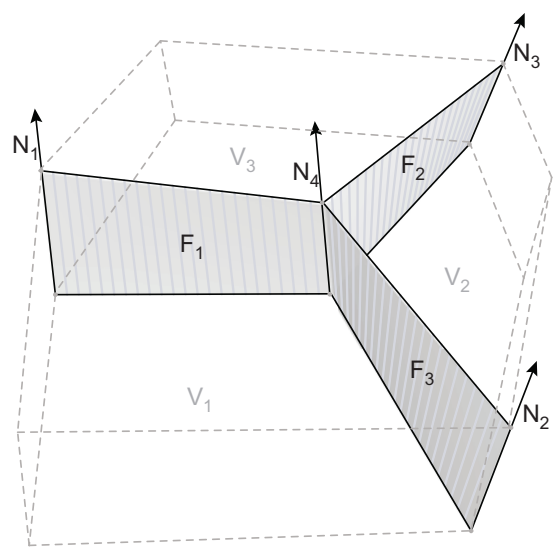

(a)

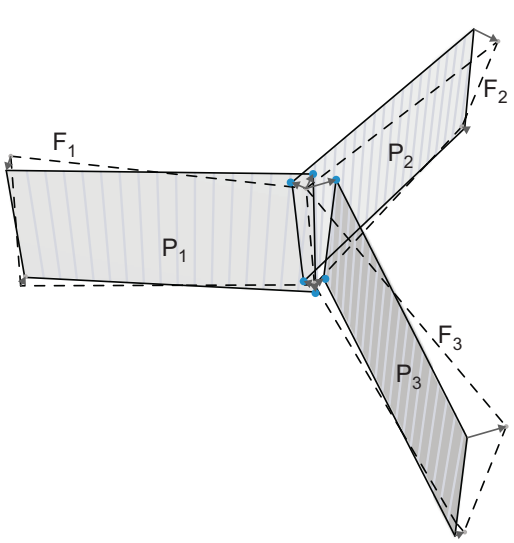

(b)

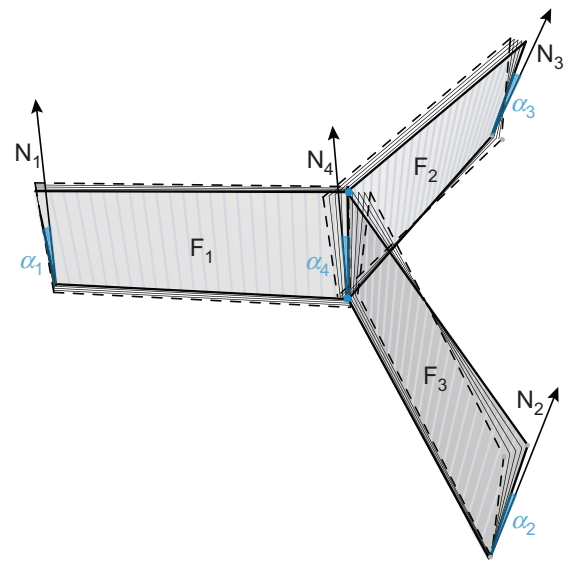

(c)
Figure 7. (a) The initial, ruled contact faces $F_{1}, F_{2}, F_{3}$ between the voussoirs $V_{1}, V_{2}, V_{3}$ based on the local surface normals $N_{1}, N_{2}, N_{3}$, $\mathrm{N}_{4}$. (b) Nodes of the contact faces are projected to the corresponding best fit plane $P_{1}, P_{2}, P_{3}$. (c) The barycentres of corresponding projected nodes are updated repetitively until all contact faces are planar. The angles $\alpha_{1}, \alpha_{2}, \alpha_{3}$ are used to check the deviations to the local surface normals $N_{1}, N_{2}, N_{3}, N_{4}$ 


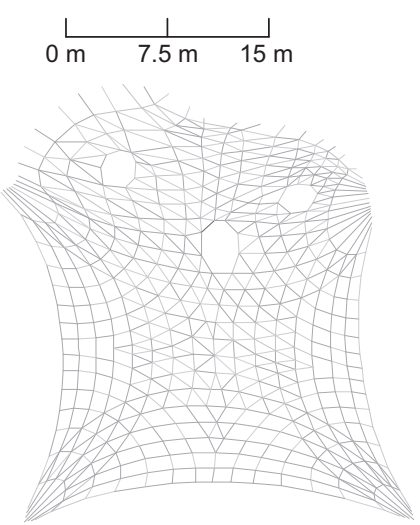

(a)
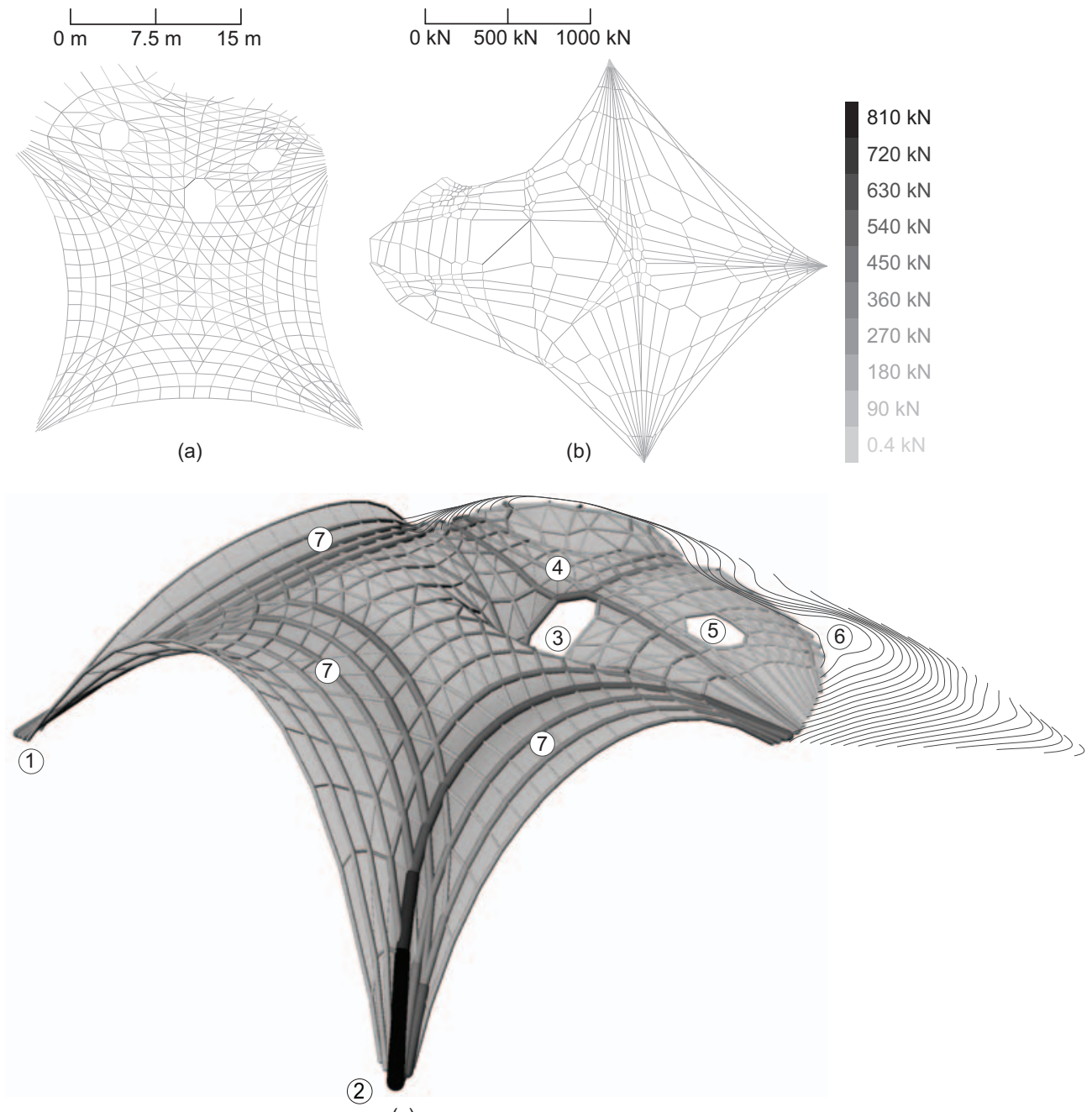

(c)

Figure 8. The TNA form-finding method, applied for the MLK Jr Park Vault: (a) the form diagram, with given scale in metres; (b) its corresponding reciprocal force diagram, with given scale in $\mathrm{kN}$; (c) the resulting thrust network; the diameter of the pipes are proportional to the thrusts in the edges; the circled numbers (1-7) highlight intended design features
Figure 8(a), summing up to a resultant thrust of $1814 \mathrm{kN}$ at that support.

Specific, intended design features are highlighted in Figure 8(c), such as two supports forming the main opening with a span of almost $28 \mathrm{~m}$ (numbers 1 and 2). This results in high horizontal forces at the supports, as visualised by the diameter of the pipes, which are proportional to the thrusts in the edges. The 'eye openings' supported in one point (numbers 3 and 4) are formally inspired by the iconic design features of several of Frei Otto's cable-net structures, for example the German Pavilion at Expo 1967, Montreal, Canada (Sitte et al., 1967). During the form finding, when only self-weight was considered, these point supports have only vertical reaction forces to the ground; all horizontal forces in that point are balanced in the structure, as shown by the balanced force diagram. Additional features are the oculus (number 5) and the raised curved support forming a smooth transition to the surrounding topography (number 6). Through manipulation of the force diagram (Figure 8(b)) (see Section 3.1), proportionally more forces have been attracted parallel to the forces in the outer edges, resulting in the 'flaringup' edge arches (Figure 8(c), number 7). These form an opening gesture towards the surrounding park, and are reminiscent of some of the shells of Heinz Isler, for example the Naturtheater Grötzingen shell in Grötzingen, Germany (Chilton, 2000).

These examples of features, even though some of them reference existing cable-net and concrete shell structures, show 
a novel, formal and structural language for unreinforced masonry vaults.

\subsection{Tessellation}

Figure 9 shows the user-defined tessellation pattern after the alignment to the force flow.

Fabrication and assembly constraints were taken into account by limiting the length of specific edges. The maximum and minimum size of resulting voussoirs and the interlocking dovetail shape were controlled during the design process. Using the assisted and informed design process presented in Section 3.2, it was possible to generate the tessellation consisting of 734 polygons on the complex target surface. Particularly the high number of singularities of the force vector field caused many topological changes of the generic tessellation geometry, as shown in Figure 6. Generating the tessellation using a manual design process, considering all interdependent constraints described, would have been very tedious for a structure of this size and complexity, if not impossible.

\subsection{Voussoir geometry}

In contrast with reinforced concrete shells (e.g. Service Station Deitlingen Süd, Switzerland, 1968 by H. Isler, with an average thickness of $90 \mathrm{~mm}$ ), unreinforced, stone-cut masonry shells cannot resist bending. To resist non-funicular live load cases and avoid buckling, both the depth and the self-weight of the vault have been increased (Allen and Zalewski, 2010). The thickness of the MLK Jr Park Vault ranges therefore between 250 and $1000 \mathrm{~mm}$ based on first calculations using TNA, making its self-weight clearly a very dominant loading case.

The contact faces between the voussoirs are all planar, as required by the fabrication constraints (Section 2.5). The

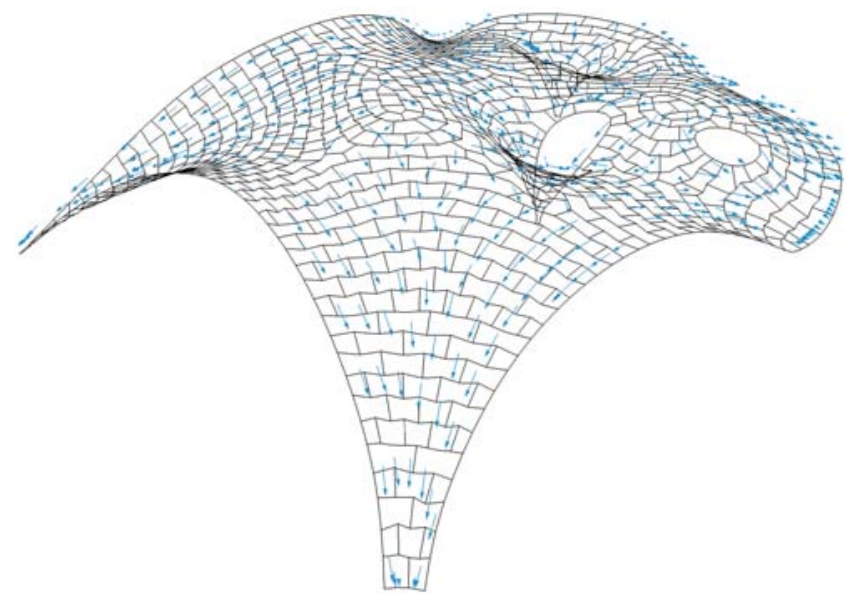

Figure 9. Resulting tessellation considering the designed flow of forces planarisation of all contact faces was realised using the optimisation process in Section 3.3. Figure 10 visualises the distribution of deviation angles for all edges. For $20 \%$ of the, in total, 1599 edges, the deviation angle is less than $1^{\circ}$, and for more than two-thirds of the edges the deviation angle is less than $5^{\circ}$. Just a few edges at locations with high surface curvature (Figure 8(c), numbers 3 and 4 ) have higher deviation angles; this means that neighbouring contact faces might not be sufficiently aligned to the force flow. In these areas all faces need to be carefully checked for possible sliding failure. Additionally, one could exclude these faces from the planarisation procedure, maintaining the ruled-surface geometry, and locally use alternative manufacturing techniques (e.g. multiaxes milling) for the fabrication of the affected voussoirs in those 'problem' areas. Globally, the planarisation algorithm resulted in a relatively low average deviation angle of $4.41^{\circ}$, representing a promising outcome considering the overall efficiency of the fabrication process.

\subsection{Machining strategy}

Because of the planar contact faces, saw blade cutting was selected to process the contact faces. This represents a great benefit because the blocks can be processed relatively fast with adequate precision. CNC machines, capable of handling blocks of this size, can be used to process the voussoirs (Figure 11). The machining process starts with the (virtual) alignment of the voussoir geometry within the designated stone block (Figure 11(a)). The upper and lower surfaces are approximated by parallel cuts (Figure 11(b)). This approximation is needed because the upper and lower surface of each voussoir is doubly curved. Generally, the block needs to be flipped and rereferenced in order to process both sides. Subsequently, the planar voussoir contact faces can be cut precisely by using the same machine and tool (Figure 11(c)). The waste material on the top and bottom can be removed by manual chiselling (Figure 11(d)). A further smoothening process is optional and depends on the desired surface quality. Part of the MLK Jr Park Vault will cover stage areas with high requirements for acoustics, for which a rougher surface for acoustic sound diffusion might be beneficial. In this design phase of the project, first stone voussoirs have been cut to start adjusting and refining the design and materialisation process (Figure 11(e)).

\subsection{Structural model}

Structural models allow for a first validation of and insight into structural behaviour, particularly in the case of such free-form vaults. Furthermore, owing to the scalability of compressiononly masonry structures (Block et al., 2010b; Heyman, 1995), they enable a reliable prediction of the stability of a real-scale stone structure for corresponding load assumptions. A first structural model of the MLK Jr Park Vault was built to scale 1:33 using rapid prototyping techniques (Figure 12). All 


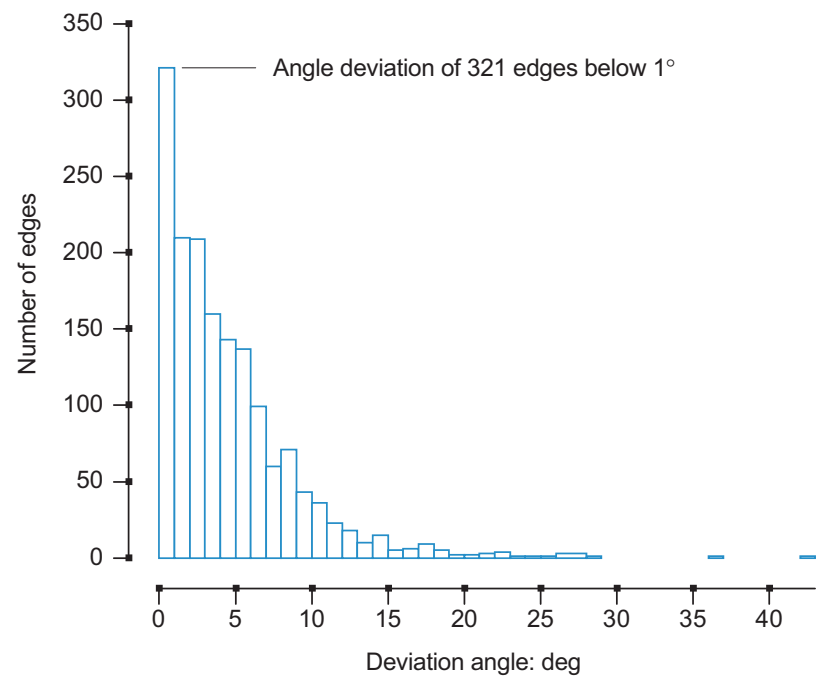

Figure 10. Resulting distribution of deviation angles after planarisation of all contact faces have an average deviation angle of $4.41^{\circ}$

voussoirs were printed as discrete pieces using three-dimensional printing technology (Van Mele et al., 2012), and using a CNCmilled foam formwork, they were assembled without the use of adhesive. Small, coloured notches were applied as labelling for the pieces and to geometrically register neighbouring voussoirs. The model was used to explore the architectural quality of the vault, but also to investigate its stability and understand collapse mechanisms of the discrete voussoirs under different support displacements and concentrated live loads (Figure 12(c)) (see www.vimeo.com/46222727). The model was also instrumental in examining strategies for decentring.

\subsection{Datasheet MLK Jr Park Vault}

Table 1 provides a summary of relevant information about the MLK Jr Park Vault according to the current design of the project.

\section{Conclusion and future research}

This paper has shown the potential of new applications of structural masonry by identifying efficient techniques for the development of discrete, free-form stone vaults. The digital design and materialisation chain for the development of these structures, allowing innovative architectural applications for a traditional material, has been discussed. The interdependent constraints, in particular structural and fabrication requirements, have been discussed and associated with basic geometric constraints. Optimisation algorithms that deal with these constraints have been developed. The techniques for tessellation and for generation and optimisation of vousoirs have been integrated in the TNA-based form-finding tool RhinoVault, which was used to design and build a detailed structural model of the MLK Jr Park Vault in Austin, Texas, USA. The result shows the efficient combination and integration of construction material and structural form; and, in the opinion of the authors, indeed, the elegance of structures that 'resist through form'.

Future research will focus on the optimisation techniques in the design process. In particular, the tessellation strategies need to be investigated further, allowing for fewer dependencies on predefined topologies to better incorporate architectural

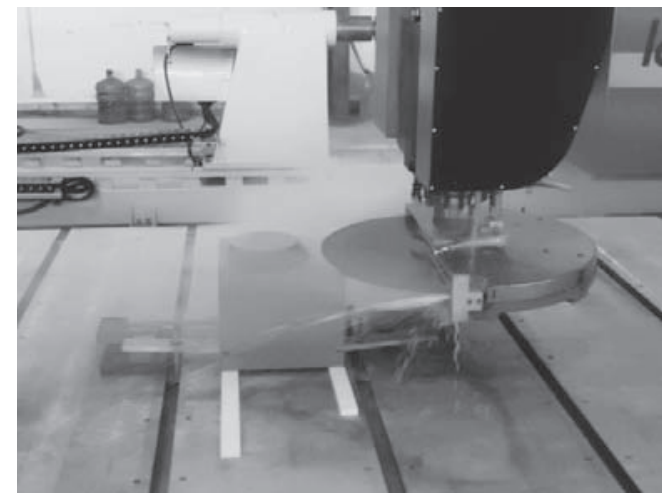

(e)

effectively by manual chiselling; (e) first voussoir prototype for the MLK Jr Park Vault, processed on a five-axes CNC stone-cutting machine, using a circular blade, at 5 Axis, LLC, Buda, Texas

(photograph by Escobedo Construction)
Figure 11. Machining strategy: (a) voussoir geometry within the stone block; (b) approximation of the upper and lower surface using parallel planar cuts; (c) planar cuts for the voussoir contact faces; (d) waste material on the top and bottom gets removed

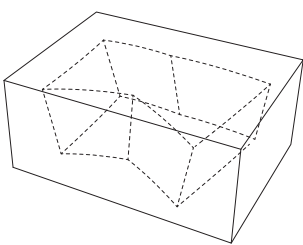

(a)

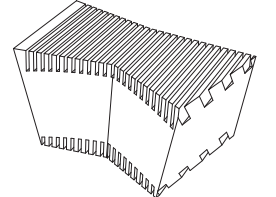

(c)

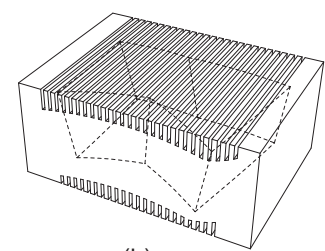

(b)

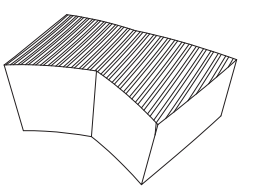

(d) 


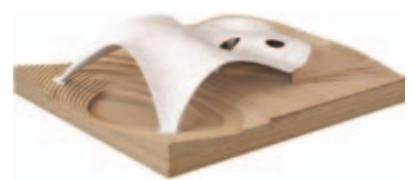

(a)

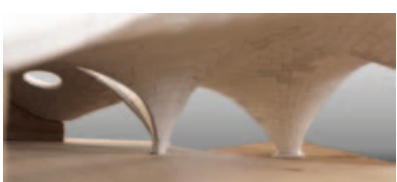

(b)

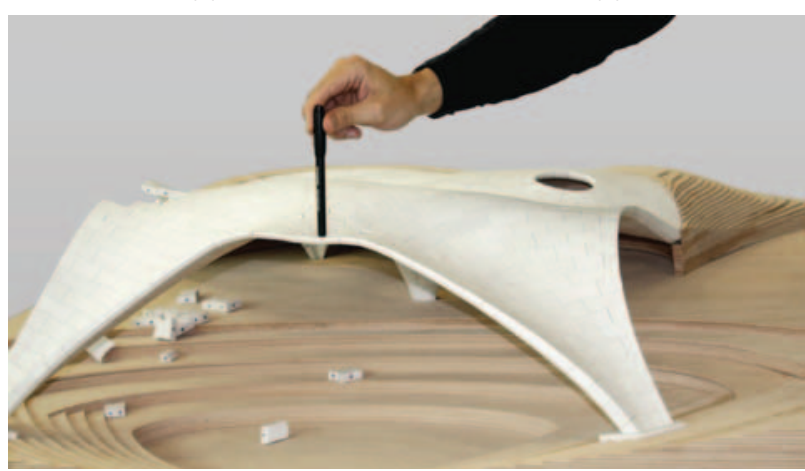

(c)

Figure 12. (a), (b) 3D-printed structural model, consisting of separate voussoir pieces, of the MLK Jr Park Vault Project (scale $1: 33) ;(c)$ partial collapse of the structural model

considerations. Another challenge and additional focus of research will be the assembly logic by identifying geometrically feasible and structurally stable construction sequences during incomplete phases of free-form vaults, which goes along with the further investigation of advanced structural analysis and efficient formwork systems.

\section{Acknowledgements}

The authors would like to thank Carmen Castanos, Anna Falvello Tomas, Ramon Weber and Marcel Aubert for their

Total surface area

Total covered area

Total number of voussoirs

Average size of voussoirs

Maximum thickness

Minimum thickness

Maximum span

Total surface area of contact faces

(planar cuts)

Total surface area of upper and lower

faces (double curved surface milling)

Volume stone

Total weight

Specific weight of Texas limestone

Table 1. Key facts and properties of the current design of the MLK Jr Park Vault help building and testing the structural model, Lorenz Lachauer for his input in the initial design stage of the project, and Dr Tom Van Mele for his patient editing.

The authors are also thankful for the opportunity to be part of the development team of the MLK Jr Park Vault in Austin, Texas, USA. Client and development partner: MFI Real Estate, LLC; general contractor: Escobedo Construction, LP; stone fabrication and installation: Escobedo Construction, LP and 5 Axis, LLC; structural design: BLOCK Research Group, ETH Zurich, Switzerland; structural engineering: Ochsendorf, DeJong \& Block, LLC.

\section{REFERENCES}

Allen E and Zalewski W (2010) Form and Forces. John Wiley and Sons, New York, NY, USA.

Bechthold M (2009) New stone shells: design and robotic fabrication. Proceedings of the 50th International Association for Shell and Spatial Structures Symposium, Valencia, Spain, pp. 1780-1789.

Block P (2009) Thrust Network Analysis: Exploring Threedimensional Equilibrium. Department of Architecture, Massachusetts Institute of Technology, Cambridge, MA, USA.

Block P and Lachauer L (2011) Closest-fit, compression-only solutions for free form shells. Proceedings of the IABSEIASS Symposium 2011, London, UK, pp. 108.

Block P and Ochsendorf J (2007) Thrust network analysis: A new methodology for three-dimensional equilibrium. Journal of the IASS 48(3): 167-173.

Block P, DeJong M, Davis L and Ochsendorf J (2010a) Tile vaulted systems for low-cost construction in Africa. Journal of the African Technology Development Forum 7(1-2): 4-13.

Block P, Lachauer L and Rippmann M (2010b) Validating thrust network analysis using 3D-printed, structural models. Proceedings of the 51st International Association for Shell and Spatial Structures Symposium, Shanghai, China, pp. 1086-1095.

Chilton JC (2000) The Engineer's Contribution to Contemporary Architecture: Heinz Isler. Thomas Telford, London, UK.

Davis L, Rippmann M, Pawlofsky T and Block P (2012) Innovative funicular tile vaulting: A prototype in Switzerland. The Structural Engineer 90(11): 46-56.

DeJong MJ (2009) Seismic Assessment Strategies for Masonry Structures. Department of Architecture, Massachusetts Institute of Technology, Cambridge, MA, USA.

Dieste E (1996) Architecture and construction. In Eladio Dieste 1943-46 (Jiménez A (ed.)). Conserjeria Obras Publicas y Transportes, Seville, Spain.

Fallacara G (2006) Digital stereotomy and topological transformations. Proceedings of the 2nd International Congress on Construction History, Cambridge, UK.

Fallacara, G (2009) Toward a stereotomic design: Experimental constructions and didactic experiences. Proceedings of the 
3rd International Congress on Construction History,

Cottbus, Germany, p. 553.

Fitchen J (1981) The Construction of Gothic Cathedrals: A Study of Medieval Vault Erection. University of Chicago Press, Chicago, IL, USA.

Garrido Campos J and Marín Martín R (2010) Modelling and implementing circular sawblade stone cutting processes in STEP-NC. Robotics and Computer-Integrated Manufacturing 26(6): 602-609.

Gramazio F and Kohler M (2008) Digital Materiality in Architecture. Lars Müller Publishers, Zurich, Switzerland.

Heyman J (1995) The Stone Skeleton: Structural Engineering of Masonry Architecture. Cambridge University Press, Cambridge, UK.

Kaczynski MP, McGee W and Pigram DA (2011) Robotically fabricated thin-shell vaulting: A methodology for the integration of multi-axis fabrication processes with algorithmic form-finding techniques. Proceedings of the 31st Annual Conference of the ACADIA, Calgary, Canada, pp. 114-121.

Pedersen OE, Larsen NM and Pigram D (2012) Realisation of complex precast concrete structures through the integration of algorithmic design and novel fabrication techniques. Proceedings of Advances in Architectural Geometry 2012, Paris, France.

Pigram DA and McGee W (2011) Formation embedded design: A methodology for the integration of fabrication constraints into architectural design. Proceedings of the 31st Annual Conference of the ACADIA, Calgary, Canada, pp. 122-131.

Ramage M, Ochsendorf J, Rich P, Bellamy J and Block P (2010a) Design and construction of the Mapungubwe National Park Interpretive Centre, South Africa. Journal of the African Technology Development Forum 7(1-2): 14-23.

Ramage M, Ochsendorf J and Rich P (2010b) Sustainable shells: new African vaults built with soil-cement tiles. Journal of the IASS 51(4): 255-261.

Rippmann M and Block P (2011) New design and fabrication methods for freeform stone vaults based on ruled surfaces. Proceedings of the Computational Design Modelling Symposium Berlin, Germany, pp. 181-189.

Rippmann M, Lachauer L and Block P (2012) Interactive vault design. International Journal of Space Structures 27(4): 219-230.

Sitte FM, Expo and Eisenberg G (1967) Expo'67 MontrealDeutscher Pavillon: Dokumentation über das Bauwerk. Werner, Düsseldorf, Germany (in German).

Van Mele T, Mclnerney J, DeJong M and Block P (2012) Physical and computational discrete modeling of masonry vault collapse. Proceedings of the 8th International Conference on Structural Analysis of Historical Constructions, Wroclaw, Poland.

Wendland D (2009) Experimental construction of a free-form shell structure in masonry. International Journal of Space Structures 24(1): 1-11.

\section{WHAT DO YOU THINK?}

To discuss this paper, please email up to 500 words to the editor at journals@ice.org.uk. Your contribution will be forwarded to the author(s) for a reply and, if considered appropriate by the editorial panel, will be published as discussion in a future issue of the journal.

Proceedings journals rely entirely on contributions sent in by civil engineering professionals, academics and students. Papers should be 2000-5000 words long (briefing papers should be 1000-2000 words long), with adequate illustrations and references. You can submit your paper online via www.icevirtuallibrary.com/content/journals, where you will also find detailed author guidelines. 